\title{
Influence of ionised electrons on heavy nuclei angular differential scattering cross sections
}

\author{
J Ullrich $\uparrow, \mathrm{R}$ E Olson $\ddagger$, R Dörner†, V Dangendorf $\uparrow, \mathrm{S}$ Kelbch $\uparrow$, \\ $\mathrm{H}$ Berg $\dagger$ and $\mathrm{H}$ Schmidt-Böcking $\dagger$ \\ † Institut für Kernphysik, Universität Frankfurt, D-6000 Frankfurt/Main, Federal Republic \\ of Germany \\ $\ddagger$ Physics Department, University of Missouri, Rolla, MO 65401, USA
}

Received 2 September 1988

\begin{abstract}
For the collision systems $1.4 \mathrm{MeVu}^{-1} \mathrm{U}^{32+}$ and $5.9 \mathrm{MeVu}^{-1} \mathrm{U}^{65+}$ on $\mathrm{Ne}$, transverse (with respect to the beam axis) momentum distributions of recoiling target atoms have been measured applying a time-of-flight technique. In the case of isotropic electron emission, the transverse momenta of the recoil ion $p_{\mathrm{R} \perp}$ and the projectile $p_{\mathrm{P}_{\perp}}$ after the collision are identical. This allows the transformation of measured $p_{\mathrm{R} \perp}$ distributions into projectile related differential scattering cross sections $\mathrm{d} \sigma / \mathrm{d} \theta$. Using such an analysis, we have measured differential cross sections in the scattering angle regime of $1 \times 10^{-6} \mathrm{rad} \leqslant \theta \leqslant$ $4 \times 10^{-5} \mathrm{rad}$. The shape, as well as the absolute magnitude of the derived experimental $\mathrm{d} \sigma / \mathrm{d} \theta$ is in reasonable agreement with Rutherford differential cross sections at large $\theta$ using a projectile nuclear charge of $Z_{\mathrm{P}}=q_{\mathrm{P}}$ and a target nuclear charge of $Z_{\mathrm{T}}=5$. For $\theta \leqslant 1 \times 10^{-5} \mathrm{rad}, n$-body classical trajectory Monte Carlo (nCTMC) calculations predict that the balance between $p_{R_{\perp}}$ and $p_{P_{\perp}}$ is strongly influenced by the momenta of anisotropically emitted ionised electrons. From the comparison between the derived experimental cross sections $\mathrm{d} \sigma / \mathrm{d} \theta$ with the theoretical values, as well as from the agreement between experimental and theoretical transverse recoil-ion momentum distributions, we conclude that the momenta of the ejected electrons have a considerable influence on the scattering dynamics of the heavy nuclei. Furthermore, the calculations indicate that the projectile is scattered to negative angles at impact parameters of $b \geqslant 3 a_{0}$ due to anisotropic electron emission and strong polarisation of the target electron cloud by the Coulomb potential of the projectile. The possibility of rainbow scattering at $\theta=1 \times 10^{-6} \mathrm{rad}$ is predicted.
\end{abstract}

\section{Introduction}

The knowledge of angular differential cross sections for ion-atom single collisions is of basic interest for the understanding of phenomena such as multiple scattering, beam transport applications in accelerators, sputtering, and ion implantation, as well as for the theoretical description of particle range and stopping power.

Several collections of experimental investigations of angular differential scattering for slow and highly charged projectiles impinging on gaseous targets can be found in the literature for projectile energies up to $200 \mathrm{keV} \mathrm{u}^{-1}$ (see, e.g., Proceedings of the Conference on the Physics of Multiply Charged Ions 1987). Only a few measurements of differential cross sections have been reported in the medium-energy regime $E_{\mathrm{P}} \leqslant$ $10 \mathrm{MeVu}^{-1}$ (Schuch et al 1988, Ullrich et al 1988a, Kelbch et al 1988) due to the problem of detecting projectile scattering at very small angles. 
In this paper we report experimental data on absolute angular differential scattering cross sections in the medium-energy regime $\left(1.4\right.$ and $\left.5.9 \mathrm{MeV} \mathrm{u}^{-1}\right)$ for highly-charged uranium ion impact on a $\mathrm{Ne}$ gas target in an angular range of $1 \times 10^{-6} \mathrm{rad} \leqslant \theta \leqslant$ $4 \times 10^{-5} \mathrm{rad}$. These data are of particular importance for beam transport in heavy-ion, high-energy storage rings. Our experimental procedure ensures single-collision conditions. Also, since the incoming projectile charge state was varied from $32+$ to $65+$, a strong projectile charge dependence in the cross sections could be observed.

From the comparison of the experimental data with theoretical results in a recently developed nCTMC approach, we conclude that for the smallest scattering angles, inelastic processes such as electron emission and polarisation of the target electron cloud by the strong incoming Coulomb field have considerable, if not decisive, influence on the dynamics of the collision process and on measured differential scattering cross sections. This is in striking contrast to previous theoretical approaches, where inelastic electronic processes are not related to the heavy particle scattering. The calculations show that all the particles, including the target electrons, have to be considered in order to describe accurately the dynamics of high-energy, highly-charged, heavy-ionrare-gas collisions.

\section{Theory}

The theoretical calculations were conducted using the $n$-body classical trajectory Monte Carlo method $(n \mathrm{CTMC})$. This is a three-dimensional approach that includes not only the motion of the heavy nuclei, but also explicitly incorporates the motion of all the target electrons. The projectile is assumed to be a point charge which is a valid approximation for these calculations since the projectile stripping cross section is known to be several orders of magnitude less than that for target ionisation. All Coulomb interactions are included except those between individual electrons. Hence, electron correlation effects will not be elucidated, but recoil and momentum transfer processes between the heavy nuclei and the electrons are incorporated. The classical Hamiltonian and the coupled equations that are numerically solved have been given previously (Olson 1988). It should be noted that for collisions such as those studied here, the nCTMC method has successfully predicted the collision dynamics of target recoil-ion energies (Olson et al 1987b), projectile scattering and inelasticities (Schuch et al 1988), and ionised electron angular and energy distributions (Schmidt-Böcking et al 1988). In this paper we will demonstrate the importance of the ejected electrons in determining the angular deflections of the heavy nuclei.

For the reactions under consideration here, $1.4 \mathrm{MeVu}^{-1} \mathrm{U}^{32+}+\mathrm{Ne}$ and $5.9 \mathrm{MeV} \mathrm{u}^{-1} \mathrm{U}^{65+}+\mathrm{Ne}$, it was necessary to compute 40000 individual trajectories for each reaction in order to obtain the differential cross sections. Seventy two coupled, first-order differential equations are solved for each trajectory so that the $x y z$ momenta and positions of each particle could be followed throughout the collision. The initial electron distributions were determined from spectroscopic energy levels. No adjustable parameters are used in order to obtain the absolute differential cross sections.

\section{Experiment}

The experiments were performed at the UNILAC accelerator of GSI, Darmstadt. The 1.4 $\mathrm{MeV} \mathrm{u}^{-1}$ data were obtained in the GSI 'Stripperhalle' 
Since the scattering angles of interest are on the order of microradians for the above collision systems, their conventional determination, namely measuring the deflection of the projectile from the beam axis at a certain distance apart from the collision region, fails. According to the detailed descriptions given elsewhere (Ullrich et al $1988 \mathrm{~b}$ ), this was the motivation to develop a new time-of-flight spectrometer (TOFs), which determines the recoil-ion momentum $p_{\mathrm{R} \perp}$ perpendicular to the beam axis instead of that of the projectile $p_{P_{\perp}}$ (for a small projectile laboratory scattering angle $\theta$ one obtains the identity $\theta=\left|\boldsymbol{p}_{\mathrm{P} \perp}\right| /\left|\boldsymbol{p}_{0}\right|$, where $\boldsymbol{p}_{0}$ is the momentum of the incoming projectile). As is illustrated in figure 1 , a momentum diagram of the collision in the laboratory frame, both transverse momentum components have the same magnitude $\left|p_{\mathrm{R} . \perp}\right| \approx\left|\boldsymbol{p}_{\mathrm{P} \perp}\right|$ if the sum of all electron momenta $\Sigma \boldsymbol{p}_{\mathrm{e} \perp}$ is equal to zero. Until now, there has been no information from experimental data or theoretical calculations that the target electron emission could be anisotropic with respect to the azimuthal angle for a fixed scattering plane. Hence, it has been assumed that $\Sigma p_{e_{\perp}}$ is equal to zero. Under this assumption, measured $p_{\mathrm{R} \perp}$ distributions can be transformed into $\mathrm{d} \sigma / \mathrm{d} \theta$ (for details see Ullrich et al (1988b)) according to (1) for small projectile scattering angles $\theta$ :

$$
\theta=\left|p_{\mathrm{R} \perp}\right| /\left(2 E_{\mathrm{P}} M_{\mathrm{P}}\right)^{1 / 2} .
$$

In equation (1), $M_{\mathrm{P}}$ is the projectile mass and $E_{\mathrm{P}}$ the projectile energy. Even if this identity does not hold due to anisotropic electron emission during the encounter $\left(\Sigma \boldsymbol{p}_{\mathrm{e} \perp} \neq 0\right)$ the information derived about the collision process is equivalent to that of measuring the projectile scattering.



Figure 1. Momentum diagram of the collision in the laboratory frame of reference. $p_{0}$, incoming projectile momentum; $\boldsymbol{p}_{\mathrm{P} \perp}$, scattered projectile transverse momentum; $\boldsymbol{p}_{\mathrm{R} \perp}$, target atom transverse momentum; $\Sigma \boldsymbol{p}_{\mathrm{e} \perp}$, sum of transverse electron momenta.

The recoil-ion transverse momentum is obtained by applying a time-of-flight technique: a drift path 'free' of electric and magnetic fields with a fixed length $(5 \mathrm{~mm})$ is provided, which is travelled by the recoiling target ion in a time interval proportional to its transverse momentum gained by the collision. The coincidence between the signal of the recoil ion and the delayed signal of the projectile gives the time-of-flight (TOF) information. The measured TOF has to be corrected to account for the flight times of the projectile from the collision region into the detector, for the TOF of the recoil ion inside the detection branch of the apparatus (without the drift path), and for electronic processing times.

A charge state analysed ( 1 in figure 2 ) uranium beam of the GSI UNILAC was collimated (2) to a beam spot of less than $0.2 \times 0.2 \mathrm{~mm}^{2}$, the last collimator being placed only about $8 \mathrm{~cm}$ in front of the TOFS (5). Thus the 'starting point' of the recoil ions within the field free region of the TOFs (9) was well defined. The recoil ions were charge state analysed in a magnetic field (7) and detected (6) as described in Ullrich 


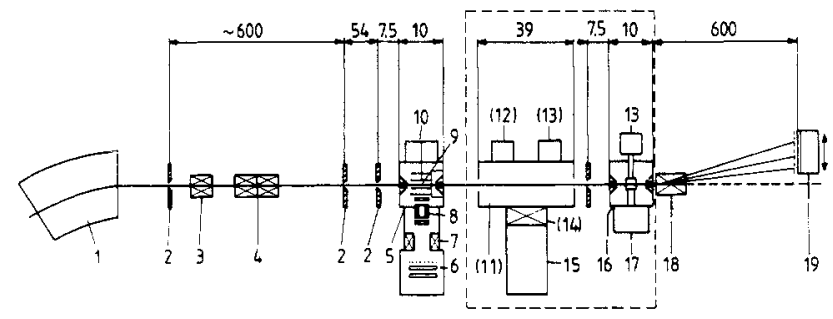

Figure 2. Experimental set-up (all distances given on the figure are in $\mathrm{cm}$ ). 1, magnet; 2, collimator; 3 , steerer; 4 , quadrupole lens; 5 , recoil-ion momentum spectrometer; 6 , channelplate detector; 7 , analysing magnets; 8 , einzel lens; 9 , scattering region; 10 , gas inlet; 11 , charge exchange region; 12, viscosity pressure manometer; 13 , capacitance manometer; 14 , valve; 15 , vacuum pump; 16 , three-stage differential pumping system; 17 , recoil-ion time-of-flight spectrometer; 18 , analysing magnet; 19 , parallel-plate avalanche detector.

et al (1988b). For the case of $5.9 \mathrm{MeV} \mathrm{u}^{-1} \mathrm{U}^{65+}$ on $\mathrm{Ne}$, the projectiles having passed the collision region entered another, conventional recoil spectrometer (17) (for details see Kelbch et al (1984)), where the ions were extracted out of the target region by an electric field. In this spectrometer the recoil-ion charge state analysis was performed taking advantage of the fact that their ToF within the accelerating electric field depends on the charge state of the created ions. The reason for mounting a second spectrometer was to control the efficiency of the TOFs in an independent way. This part of the apparatus (surrounded by the broken line in figure 2) was omitted in the $1.4 \mathrm{MeV} \mathrm{u}^{-1}$ experiment, since the efficiency of the apparatus could be shown to be independent of the recoil-ion charge state by the comparison with experimental data from Schuch (Ullich et al 1988a). Finally, in both experiments the projectiles were charge state analysed in a magnetic field (18) and all final charge states were detected simultaneously by a position sensitive parallel plate avalanche detector (19).

The pressure inside the whole beamline was less than $10^{-6}$ Torr for both experiments; the target pressure in both spectrometers was about $10^{-4}$ Torr. Differential pumping around each target region ensured that the target was well localised and therefore the initial and final projectile charge states are accurately defined.

Absolute charge-changing cross sections were derived from the pressure dependence of charge exchanged fractions of the incoming beam. These data were obtained in separate experiments for both collision systems. For this purpose an extended $(39 \mathrm{~cm}$ long), differentially pumped, gas cell was provided between the two spectrometers (11). The pressure was regulated by a servo leak valve and measured by a capacitance manometer (13), and additionally, by a viscosity pressure manometer (12).

\section{Data analysis}

\subsection{Relative differential scattering cross sections}

As mentioned above, the measured $p_{\mathrm{R} \perp}$ distributions for each recoil-ion charge state $q_{\mathrm{R}}$ and final projectile charge state $q_{\mathrm{P}}$ were converted into differential cross sections with respect to the relative momentum transfer to the recoil ion $\mathrm{d} \sigma\left(q_{\mathrm{R}}, q_{\mathrm{P}}\right) / \mathrm{d}\left(p_{\mathrm{R} \perp} / p_{0}\right)$, where $p_{\mathrm{R} \perp}=\left|\boldsymbol{p}_{\mathrm{R} \perp}\right|$ and $p_{0}=\left|\boldsymbol{p}_{0}\right|$. Assuming isotropic electron emission $\left(\Sigma \boldsymbol{p}_{\mathrm{e} \perp}=0\right)$ this quantity equals the angular differential cross section $\mathrm{d} \sigma\left(q_{\mathrm{R}}, q_{\mathrm{P}}\right) / \mathrm{d} \theta$. The total relative 
differential scattering cross section for ionisation $\mathrm{d} \sigma / \mathrm{d}\left(p_{\mathrm{R} \perp} / p_{0}\right)$ could be obtained by summation over all reaction chanxels $q_{\mathrm{R}}$ and $q_{\mathrm{p}}$ :

$$
\mathrm{d} \sigma / \mathrm{d}\left(p_{\mathrm{R} \perp} / p_{0}\right)=\sum_{q_{\mathrm{R}}, q_{\mathrm{P}}} \mathrm{d} \sigma\left(q_{\mathrm{R}}, q_{\mathrm{P}}\right) / \mathrm{d}\left(p_{\mathrm{R} \perp} / p_{0}\right) .
$$

The analysis of the experimental cross sections $\mathrm{d} \sigma\left(q_{\mathrm{R}}, q_{\mathrm{P}}\right) / \mathrm{d}\left(p_{\mathrm{R} \perp} / p_{0}\right)$ (Ullich et al 1988a) in the framework of the independent particle model (IPM, McGuire and Weaver $1977)$ assuming $\theta \approx\left(p_{R_{\perp}} / p_{0}\right)$ yielded projectile scattering angle dependent multiple ionisation probabilities $P^{q_{R}}(\theta)$. In accordance with theoretical results in the semiclassical quantum statistical approach (Horbatsch 1986), the total ionisation probability $P_{\mathrm{T}}(\theta)$,

$$
P_{\mathrm{T}}(\theta)=\sum_{q_{\mathrm{R}}} P^{q_{\mathrm{R}}}(\theta)
$$

is to a good approximation unity in the scattering angle (or relative momentum transfer) regime of $\theta \geqslant 4 \times 10^{-6} \mathrm{rad}$ for $1.4 \mathrm{MeVu}^{-1} \mathrm{U}^{32+}$ on $\mathrm{Ne}$ and $\theta \geqslant 2 \times 10^{-6} \mathrm{rad}$ for $5.9 \mathrm{MeV} \mathrm{u}^{-1} \mathrm{U}^{65+}$ on $\mathrm{Ne}$. Due to the strong perturbation by the Coulomb force of the projectile's incident charge state, the probability that the target atom will not be ionised at small impact parameters when the projectile scatters to angles $\theta$ larger than the limits mentioned above is essentially zero. Therefore, the total differential scattering cross section $\left(\mathrm{d} \sigma / \mathrm{d}\left(p_{\mathrm{R} \perp} / p_{0}\right)\right)^{\mathrm{T}}$ is approximately identical to the measured one:

$$
\left(\mathrm{d} \sigma / \mathrm{d}\left(p_{\mathrm{R} \perp} / p_{0}\right)\right)^{\mathrm{T}} \approx \mathrm{d} \sigma / \mathrm{d}\left(p_{\mathrm{R} \perp} / p_{0}\right) \text {. }
$$

Furthermore, for smaller $\left(p_{\mathrm{R} \downarrow} / p_{0}\right)$ the fraction of recoil ions being scattered with $\left(p_{R \perp} / p_{0}\right)$ and not being ionised could be estimated in the framework of the IPM from the calculated total ionisation probabilities: e.g., if $P_{\mathrm{T}}\left(p_{\mathrm{R} \perp} / p_{0}\right)$ is 0.1 the total differential cross section is

$$
\left(\mathrm{d} \sigma / \mathrm{d}\left(p_{\mathrm{R} \perp} / p_{0}\right)\right)^{\mathrm{T}} \approx 9\left(\mathrm{~d} \sigma / \mathrm{d}\left(p_{\mathrm{R} \perp} / p_{0}\right)\right) .
$$

As is obvious (crosses in figure 3 ), this theory-dependent correction for elastic scattering occurs only at $\left(p_{\mathrm{R} \perp} / p_{0}\right) \leqslant 4 \times 10^{-6} \mathrm{rad}$. The theoretical results in the $n$ CTMC approach only refer to collisions where the target atom was ionised.

\subsection{Absolute normalisation}

The relative $\mathrm{d} \sigma / \mathrm{d}\left(p_{\mathrm{R} \perp} / p_{0}\right)$ were obtained from the procedure described above. The absolute total electron capture cross sections have been determined from the pressure dependence of ratios of charge exchanged projectiles to the total number of incoming particles for both collision systems. From this cross section and the ratio of coincidence events in the capture channel to the ionisation channel, absolute ionisation cross sections $\sigma_{1}$ were derived (for details see Kelbch et al (1987)). Due to the identity

$$
\sigma_{\mathrm{I}}=\int_{0}^{\infty}\left(\mathrm{d} \sigma / \mathrm{d}\left(p_{\mathrm{R} \perp} / p_{0}\right)\right) \mathrm{d}\left(p_{\mathrm{R} \perp} / p_{0}\right)
$$

the $\mathrm{d} \sigma / \mathrm{d}\left(p_{\mathrm{R} \perp} / p_{0}\right)$ could be absolutely normalised.

\subsection{Experimental error}

The statistical errors are indicated in the experimental results for $1.4 \mathrm{MeV} \mathrm{u}^{-1} \mathrm{U}^{32+}$ and $5.9 \mathrm{MeV} \mathrm{u}^{-1} \mathrm{U}^{65+}$ on $\mathrm{Ne}$. 
In the discussion given by Ullrich et al (1988b) a maximum absolute error of less than $13 \%$ in the determination of the relative transverse recoil-ion momentum $\left(p_{\mathrm{R} \perp} / p_{0}\right)$ has to be considered. Furthermore, a systematic shift of the $\left(p_{\mathrm{R} L} / p_{0}\right)$-scale of not more than $+2 \times 10^{-6}\left(1.4 \mathrm{MeV} \mathrm{u}^{-1}\right)$ and $+1 \times 10^{-6}\left(5.9 \mathrm{MeV} \mathrm{u}^{-1}\right)$ cannot be excluded.

The absolute normalisation for the 1.4 and $5.9 \mathrm{MeVu}^{-1}$ data has an estimated uncertainty of $\pm 30 \%$, due to errors in the pressure measurement and uncertainty in the determination of the effective target length.

For the collision system $5.9 \mathrm{MeV} \mathrm{u}^{-1} \mathrm{U}^{65+}$ on $\mathrm{Ne}$, the relative yield of recoil ions of specific charge states determined by the ToFs differed from the ones measured with the conventional recoil-ion spectrometer by up to $50 \%$. The deviation is most likely due to different detection efficiencies of the channel plate for different recoil-ion charge states. Since the shape of the $\left(p_{R_{\perp}} / p_{0}\right)$ distributions is not influenced by this difference, the relative yields can be taken from the data obtained with the conventional spectrometer. According to the discussion given previously, this deviation can also stem from poor efficiency of the TOFS for the combinations of small $p_{R_{\perp}}$ and simultaneously high $Q$-values (inelastic energy losses), which would also influence the shape of the TOF distributions.

The maximum error in the shape of $\mathrm{d} \sigma / \mathrm{d}\left(p_{\mathrm{R}_{\perp}} / p_{0}\right)$ due to this, which should be only of importance at very small $p_{R_{\perp}}$, could be estimated by comparing the shapes of $\mathrm{d} \sigma / \mathrm{d}\left(p_{\mathrm{R} \perp} / p_{0}\right)$ for both relative yields of recoil-ion charge states measured by the different methods. This uncertainty does not influence the data by more than a factor of two at small $\left(p_{\mathrm{R} \perp} / p_{0}\right)$ and is added to the statistical error in the results for the $5.9 \mathrm{MeV} \mathrm{u}^{-1}$ data presented below.

As has been discussed extensively, thermal motion of the target atoms influences the measured distributions only at $\left(p_{\mathrm{R} \perp} / p_{0}\right) \leqslant 6 \times 10^{-6}$ for $1.4 \mathrm{MeV} \mathrm{u}^{-1} \mathrm{U}^{32+}$ on $\mathrm{Ne}$ and $\left(p_{\mathrm{R} \perp} / p_{0}\right) \leqslant 3 \times 10^{-6}$ for $5.9 \mathrm{MeV} \mathrm{u}^{-1} \mathrm{U}^{65+}$ on $\mathrm{Ne}$. This influence was taken into account by folding the theoretical values with the calculated target atom thermal motion distribution.

Thus, the experimental $\mathrm{d} \sigma / \mathrm{d}\left(p_{\mathrm{R} \perp} / p_{0}\right)$ for $1.4 \mathrm{MeVu}^{-1} \mathrm{U}^{32+}$ on $\mathrm{Ne}$ at $\left(p_{\mathrm{R} \perp} / p_{0}\right) \geqslant$ $6 \times 10^{-6}$ have an absolute error of less than $30 \%$ plus statistical uncertainties. For the case of $5.9 \mathrm{MeV} \mathrm{u}^{-1} \mathrm{U}^{65+}$ on $\mathrm{Ne}$ the absolute error for $\left(p_{\mathrm{R} \perp} / p_{0}\right) \geqslant 6 \times 10^{-6}$ is similar. For smaller $\left(p_{R_{\perp}} / p_{0}\right)$ an error of not more than a factor of two is estimated.

Taking into account that the $\mathrm{d} \sigma / \mathrm{d}\left(p_{\mathrm{R} \perp} / p_{0}\right)$ could be determined over a range of more than four orders of magnitude for both collision systems, the magnitudes of the errors are negligibly small for the overall interpretation of the data. The discussion of $\mathrm{d} \sigma / \mathrm{d}\left(p_{\mathrm{R} \perp} / p_{0}\right)$ at very small $\left(p_{\mathrm{R} \perp} / p_{0}\right)$ suffers from the influence of the target thermal motion on the measured distributions. However, conclusions can be drawn by comparing with the theoretical calculations where the thermal motion has been incorporated.

\section{Results and discussion}

\subsection{Comparison with Rutherford differential cross sections}

In figure 3 absolute differential cross sections $\mathrm{d} \sigma / \mathrm{d}\left(p_{\mathrm{R}_{\perp}} / p_{0}\right)$ are presented for the collision systems $1.4 \mathrm{MeV} \mathrm{u}^{-1} \mathrm{U}^{32+}$ and $5.9 \mathrm{MeV} \mathrm{u}^{-1} \mathrm{U}^{65+}$ on $\mathrm{Ne}$. Since for isotropic electron emission one obtains $\left(p_{\mathrm{R} \perp} / p_{0}\right) \simeq \theta$, the experimental data can be compared to Rutherford differential cross sections in a laboratory scattering angle regime of $1 \times 10^{-6} \mathrm{rad} \leqslant \theta \leqslant 4 \times 10^{-5} \mathrm{rad}$ (note that all scattering angles and differential cross 




Figure 3. Experimental differential scattering cross sections $\mathrm{d} \sigma / \mathrm{d}\left(p_{\mathrm{R} \perp} / p_{0}\right)$ compared to Rutherford differential cross sections for the collision systems (a) $1.4 \mathrm{MeV} \mathrm{u}^{-1} \mathrm{U}^{32+}$ and (b) $5.9 \mathrm{MeVu}^{-1} \mathrm{U}^{65+}$ on Ne. Full curve: $Z_{\mathrm{P}}=q_{\mathrm{P}}, Z_{\mathrm{T}}=5$; broken curve: $Z_{\mathrm{P}}=92, Z_{\mathrm{T}}=10$ $\left(Z_{\mathrm{p}}, Z_{\mathrm{T}}\right.$, projectile and target nuclear charge; $q_{\mathrm{P}}$, incoming projectile charge state). Crosses in the upper figure: correction for elastic scattering, where the target atom is not ionised in the collision.

sections presented in this paper are in the laboratory frame of reference). The experimental data indicate possible oscillatory structure at large transverse momenta. However, the experimental errors are not sufficiently small to confidently conclude that such effects exist. It is possible that the structure is due to electronic subshells in the Ne target atom, which are sequentially ionised as the impact parameter decreases, corresponding to increasing transverse momentum of the recoil ion.

It is surprising that the shape of the measured $\mathrm{d} \sigma / \mathrm{d}\left(p_{\mathrm{R} \perp} / p_{0}\right)$ is qualitatively described by the calculated values over a wide angular regime and over several orders of magnitude in cross section. For both collision systems, the absolute magnitudes of the Rutherford differential cross sections agree best with experimental data for the choice of $Z_{\mathrm{P}}=q_{\mathrm{P}}$ and $Z_{\mathrm{T}}=5$ (full curves in figure 3 ). The cross sections for $Z_{\mathrm{P}}=92$, $Z_{\mathrm{T}}=10$ (broken curves in figure 3 ) lie above the $1.4 \mathrm{MeV} \mathrm{u}^{-1}$ data by a factor of 33 at large transverse momenta, and above the $5.9 \mathrm{MeVu}^{-1}$ experimental values by a factor of 8 . Both features, the similarity of shape to the Rutherford $\mathrm{d} \sigma / \mathrm{d} \theta$ as well as the dependence of the absolute magnitude on the incident projectile charge state are in striking contrast to the results at lower energies and low projectile charge states.

This behaviour, however, becomes qualitatively understandable if one looks at the $\left(p_{\mathrm{R} \perp} / p_{0}\right)$ range, where recoil ions of different charge states $q_{\mathrm{R}}$ are produced, illustrated in figure 4 for the collision system $1.4 \mathrm{MeV} \mathrm{u}^{-1} \mathrm{U}^{32+}$ on $\mathrm{Ne}$. Due to the strong Coulomb 
field of the projectile's nuclear charge, even highly charged Ne recoil ions are produced at small $\left(p_{\mathrm{R} \perp} / p_{0}\right) \simeq \theta$ (large internuclear distances) justifying the good agreement with experimental data for $Z_{\mathrm{T}}=5$. Negligibly small electron loss cross sections of the projectile and a small single electron capture cross section of $1 \%$ of the ionisation cross section make evident that the projectile electron cloud is not involved within these encounters: the projectile is acting in good approximation as a point charge of $Z_{\mathrm{P}}=q_{\mathrm{P}}$. For smaller projectile scattering angles the experimental data should definitely be below the differential Rutherford cross section with $Z_{\mathrm{T}}=5$ since here only lowcharged recoil ions are produced and the shielding of the target's nuclear charge due to the remaining electrons increases.

The influence of thermal motion of the target atoms on the measured cross sections (for $1.4 \mathrm{MeV} \mathrm{u}^{-1}$ ) is indicated in figure 5, by folding the calculated Rutherford cross section $(\mathrm{d} \sigma / \mathrm{d} \theta)_{\mathrm{Ruth}}$. Since $(\mathrm{d} \sigma / \mathrm{d} \theta)_{\text {Ruth }}$ is divergent at $\left(p_{\mathrm{R} \perp} / p_{0}\right)=0$, it has been set to zero for $\left(p_{R_{\perp}} / p_{0}\right) \leqslant 1 \times 10^{-6}$ and 'normalised' on the total experimental cross section. This refers to the unrealistic situation that the scattering potential remains unscreened even at the most distant collisions. The thermal motion correction (full curve in figure 5) therefore represents an upper limit of relative momentum transfer to the recoil ion for a two-body collision at these small projectile scattering angles (large impact parameters). The step rise of the experimental data towards small $\left(p_{\mathrm{R} \perp} / p_{0}\right)$ or, in other words, the large amount of recoil ions with high momentum at $\left(p_{\mathrm{R} \perp} / p_{0}\right) \leqslant 1 \times 10^{-5}$ is not understandable in terms of a pure (elastic or inelastic) two-body collision, even not for an unrealistic strong scattering potential.

\subsection{Comparison with nCTMC calculations}

In figure 6 the experimental $\mathrm{d} \sigma / \mathrm{d}\left(p_{\mathrm{R} \perp} / p_{0}\right)$ are shown together with theoretical angular differential cross sections for the projectile scattering $\mathrm{d} \sigma / \mathrm{d} \theta$ obtained in the $n$ CTMC approach: quantitative agreement can be observed for projectile scattering angles $\theta \geqslant 2 \times 10^{-5} \mathrm{rad}\left(1.4 \mathrm{MeVu}^{-1} \mathrm{U}^{32+}\right.$ impact) and $\theta \geqslant 1 \times 10^{-5} \mathrm{rad}\left(5.9 \mathrm{MeVu}^{-1} \mathrm{U}^{65+}\right.$ impact), where the theory is very close to the Rutherford differential cross section. This underlines the qualitative picture of the collision outlined above. Furthermore,

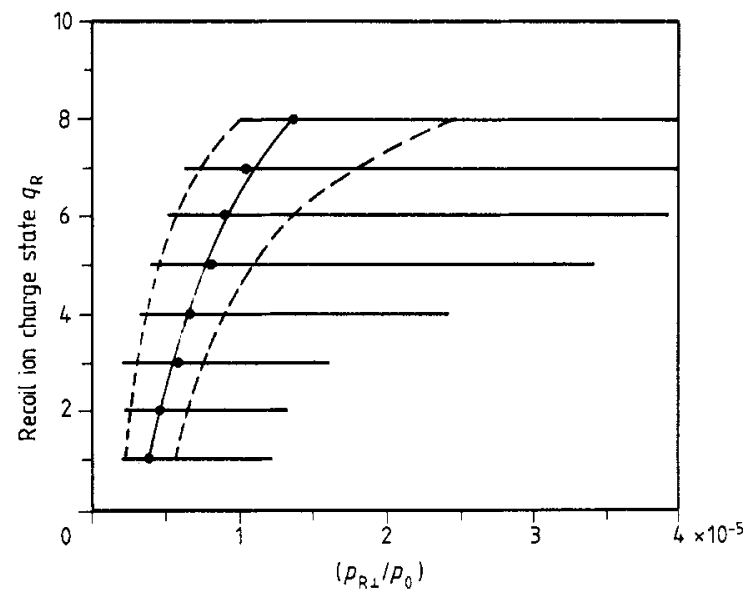

Figure 4. Relative momentum transfer distributions for the production of $q_{\mathrm{R}}$-times ionised Ne recoil ions at $1.4 \mathrm{MeV} \mathrm{u}^{-1} \mathrm{U}^{32+}$ impact. Full curve, maximum of measured distributions; Broken curves, FWHM of measured distributions. 


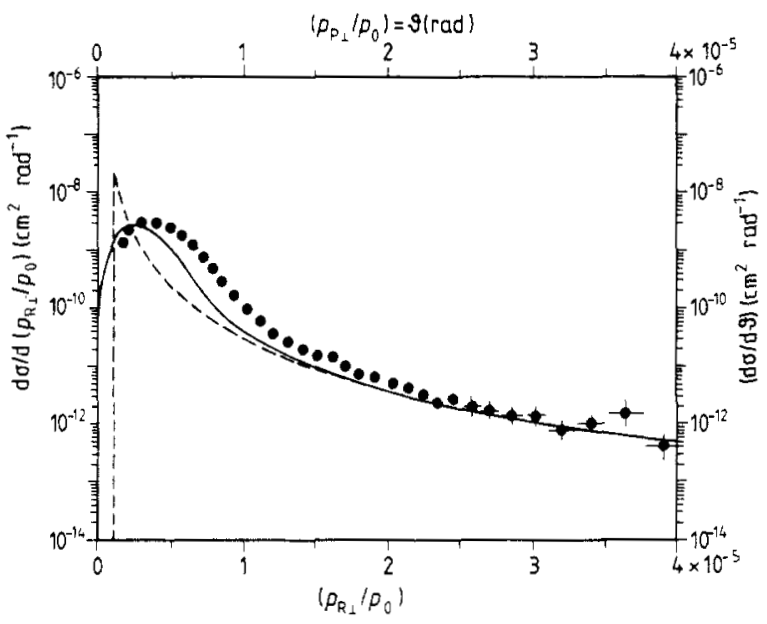

Figure 5. Calculated influence of thermal target motion on the Rutherford differential cross section for $Z_{\mathrm{P}}=q_{\mathrm{P}}, Z_{\mathrm{T}}=5$ at $1.4 \mathrm{MeV} \mathrm{u}^{-1} \mathrm{U}^{32+}$. For this comparison, the Rutherford cross sections were set equal to zero for $\left(p_{\mathrm{R}} / p_{0}\right) \leqslant 1 \times 10^{-6}$ in order to 'normalise' the theoretical values to the experimental total cross section. Broken curve, $(\mathrm{d} \sigma / \mathrm{d} \theta)_{\text {Rulh }}$; full curve, $(d \sigma / d \theta)_{R u h}$ folded with thermal motion distribution.

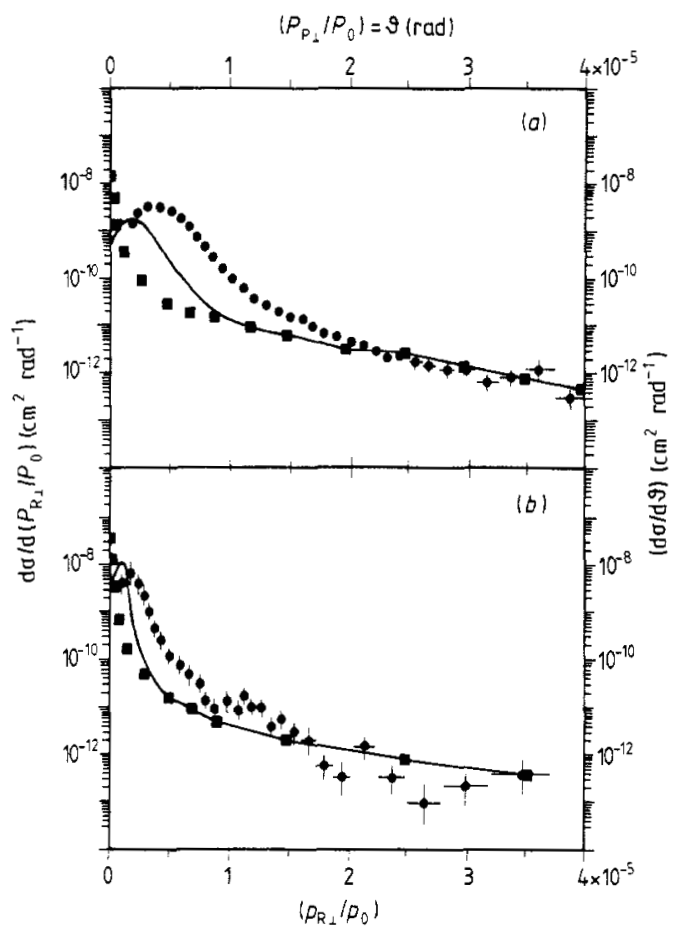

Figure 6. Experimental differential cross sections $\mathrm{d} \sigma / \mathrm{d}\left(p_{\mathrm{R} L} / p_{0}\right)$ (full circles) and theoretical (full squares) cross sections for projectile scattering $\mathrm{d} \sigma / \mathrm{d} \theta$ in collisions of (a) $1.4 \mathrm{MeVu}^{-1} \mathrm{U}^{32+}$ and (b) $5.9 \mathrm{MeVu}^{-1} \mathrm{U}^{65+}$ with $\mathrm{Ne}$. The full curve represents the theoretical values folded with the target's thermal motion distribution. 
a reasonable behaviour of the $n$ CTMC results at smaller $\theta$, namely remaining increasingly smaller than the $(\mathrm{d} \sigma / \mathrm{d} \theta)_{\text {Ruth }}$ with decreasing $\theta$, can be observed. However, these results are in disagreement with the experimental data for $\theta \leqslant 2 \times 10^{-5} \mathrm{rad}$ (1.4 $\mathrm{MeV} \mathrm{u}^{-1} \mathrm{U}^{32+}$ impact) and $\theta \leqslant 1 \times 10^{-5} \mathrm{rad}\left(5.9 \mathrm{MeV} \mathrm{u}^{-1} \mathrm{U}^{65+}\right.$ impact), as becomes evident by comparing the nстMC calculations folded with the thermal motion of the target (full curve) with the measurements.

As has been discussed in a previous paper (Olson et al 1987b), the nCTMC calculations predict, in agreement with experiment (Schmidt-Böcking et al 1988), an unexpected yield of high-energy $\delta$-electrons being emitted at about $60^{\circ}$ with respect to the beam axis. The momentum carried away by these anisotropically emitted electrons $\Sigma p_{\mathrm{e} \perp}$ should, according to the calculations, strongly disturb the identity $\left|\boldsymbol{p}_{\mathrm{R} \perp}\right| \simeq\left|\boldsymbol{p}_{\mathrm{P}_{\perp}}\right|$ at small projectile scattering angles. Additionally, the calculations show that the target electron cloud is polarised by the strong projectile Coulomb field which has a considerable influence on the dynamics of the collision process at small scattering angles.

To visualise this effect, theoretical differential cross sections for the recoil ion $\mathrm{d} \sigma / d\left(p_{\mathrm{R} \perp} / p_{0}\right)$ were calculated. A striking difference between theoretical cross sections depending on the projectile transverse momentum $\mathrm{d} \sigma / \mathrm{d}\left(p_{\mathrm{P}_{\perp}} / p_{0}\right)$ and the ones depending on the recoil-ion momentum $\mathrm{d} \sigma / \mathrm{d}\left(p_{\mathrm{R} \perp} / p_{0}\right)$ can be observed in figure 7 for projectile scattering angles $\theta \leqslant 2 \times 10^{-5} \mathrm{rad}\left(1.4 \mathrm{MeV} \mathrm{u}^{-1} \mathrm{U}^{32+}\right.$ impact) and $\theta \leqslant 1 \times 10^{-5} \mathrm{rad}$ (5.9 $\mathrm{MeVu}^{-1} \mathrm{U}^{65+}$ impact). By folding the deduced cross sections with the target's thermal motion distribution, quantitative agreement, within experimental error bars, between measured and calculated results can be observed for both collision systems over the complete $\left(p_{\mathrm{R} \perp} / p_{0}\right)$ range and over four orders of magnitude in the differential cross sections (figure 8 ).

We conclude from the above comparison that the scattering of energetic highlycharged heavy ions is considerably influenced by the target electrons at very small projectile scattering angles. This is underlined by the good agreement of recently

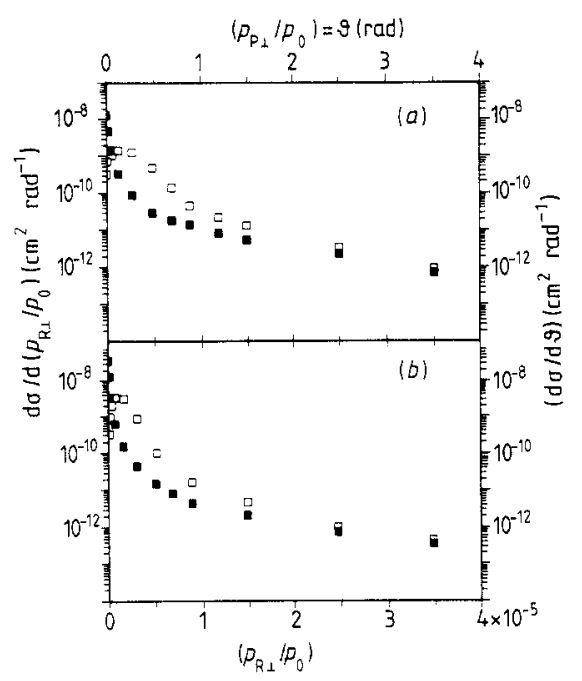

Figure 7. Theoretical differential cross sections in the nCTMC approach for the recoil ion $\mathrm{d} \sigma / \mathrm{d}\left(p_{\mathrm{R}_{-}} / p_{0}\right)$ (open squares), compared with those of the projectile $\mathrm{d} \sigma / \mathrm{d} \theta$ (full squares) for the collision systems (a) $1.4 \mathrm{MeVu}^{-1} \mathrm{U}^{32+}$ and (b) $5.9 \mathrm{MeVu}^{-1} \mathrm{U}^{65+}$ on $\mathrm{Ne}$. 


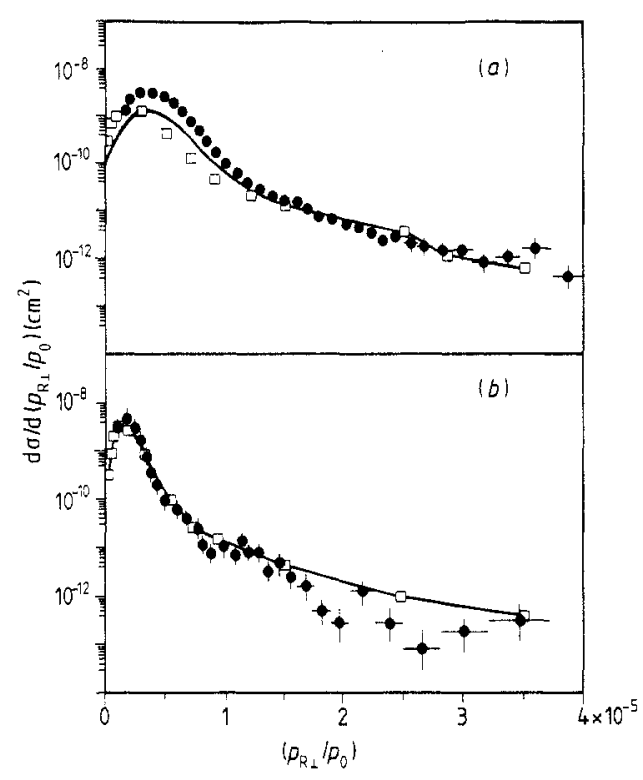

Figure 8. Recoil-ion differential cross sections: experimental (full circles) and theoretical (open squares) for the collision systems (a) $1.4 \mathrm{MeV} \mathrm{u}^{-1} \mathrm{U}^{32+}$ and (b) $5.9 \mathrm{MeV} \mathrm{u}^{-1} \mathrm{U}^{65+}$ on Ne. Full curve: theoretical results folded with thermal motion distribution.

published $\delta$-electron cross sections differential in angle and energy with the $n$ CTMC calculations (Schmidt-Böcking et al 1988).

In order to further explore the effect of polarisation of the target atom by the passage of an $\mathrm{MeVu}^{-1}$ multiply charged ion, we have numerically followed the scattering of the particles in various impact parameter ranges. It was surprising to us to find that the projectile was scattered to negative deflection angles for impact parameters greater than around $3 a_{0}$ (the impact parameter depends slightly on the recoil ion charge state). Initially, we were surprised by these results since extremely strong polarisation of the target atom is required to scatter a $0.33 \mathrm{GeV}\left(1.4 \mathrm{MeV} \mathrm{u}^{-1}\right)$ or $1.4 \mathrm{GeV}\left(5.9 \mathrm{MeV} \mathrm{u}^{-1}\right)$ uranium ion to negative deflections. Furthermore, the heavy nuclei products are positively charged, so that one would expect the strong repulsive Coulomb force of the products to dominate over any polarisation effect.

Numerical tests confirmed the attractive scattering and the following of individual trajectories elucidated the collision mechanism. Indeed we found strong polarisation of the target atom on the inward portion of the trajectory. However, most importantly, we find the polarisation is preserved on the outward track of the trajectory by the effective screening of the ionised target atom by the ejected electrons. The electrons are anisotropically ionised in the plane between the projectile and target nuclei with preferential velocities that place them between the heavy nuclei. Such ionisation events are known to dominate the ejected electron spectra for singly charged particles at low energies $\left(\mathrm{E} \sim 100 \mathrm{keV} \mathrm{u}^{-1}\right)$ and have been termed 'saddle point' ionisation (Olson et al $1987 \mathrm{a})$. The negative deflection angles only persist to small angles, $\theta \approx 1 \times 10^{-6} \mathrm{rad}$, however, the possibility of experimentally observing rainbow scattering in $\mathrm{MeV} \mathrm{u}^{-1}$ highly charged ion collisions is predicted. Such a phenomenon may be observable with modifications of the present apparatus. The importance of dynamically including the ionised electrons in the scattering of the heavy nuclei cannot be over emphasised. 
Another interesting observation arises from the calculations concerning the recoil ions. We have previously demonstrated that the $n$ CTMC method can predict the energies of the recoil ions (Olson et al 1987). However, from two-body kinematics, one would expect that the recoil ions will be emitted from the collision region at an angle of $90^{\circ}$ from the projectile ion direction. Our $n$ C TMC calculations do not confirm this expectation, but show the recoil ions preferentially scattered in a broad distribution slightly to the backward direction. For $1.4 \mathrm{MeV} \mathrm{u}^{-1} \mathrm{U}^{32+}+\mathrm{Ne}$, the maximum in the recoil ion distribution is found to be at $\sim 93^{\circ}$ relative to the projectile direction for $\mathrm{Ne}^{+}$production, with the peak position increasing to $\sim 100^{\circ}$ for $\mathrm{Ne}^{6+}$ production. Again our calculations are indicating the need to consider the momenta of the ionised electrons in the scattering dynamics of the heavy nuclei.

As an illustration of the polarisation effect, we have calculated triply differential cross sections for the $1.4 \mathrm{MeV} \mathrm{u}^{-1} \mathrm{U}^{33+}+$ Ar system (the change of target atoms from $\mathrm{Ne}$ to Ar, which is more polarisable, was necessitated by statistical error considerations within computer time restrictions). In figure 9 results are presented for $\mathrm{U}^{33+}+\mathrm{Ar} \rightarrow$ $\mathrm{U}^{33+}+\mathrm{Ar}^{4+}+4 \mathrm{e}^{-}$where the projectile is scattered to $1 \times 10^{-6} \mathrm{rad}$. The triply differential cross sections are for ionised electrons with an energy of $150 \mathrm{eV}$, where the plane of scattering is determined by the heavy nuclei. The two lobes on figure 9 represent the electron scattering for positive and negative deflection angles of $1 \times 10^{-6} \mathrm{rad}$ of the projectile. The positive angle deflections are determined in an impact parameter region around $3.6 a_{0}$, while the negative angle deflection is from $b \simeq 4.6 a_{0}$. In both cases, the electrons are ionised about the plane determined by the heavy nuclei and at a preferential direction of $\Theta_{\mathrm{e}} \approx 60^{\circ}$. Most important, however, is that the anisotropic electron emission is directed towards the projectile at its distance of closest approach. Also, the transverse momentum carried away by the electrons is greater than that of the projectile. Hence, conservation of momentum requires that the target recoil ion's transverse momentum will not equal that of the projectile. Such a conclusion is clearly demonstrated from the analysis of the experimental data given in this paper.

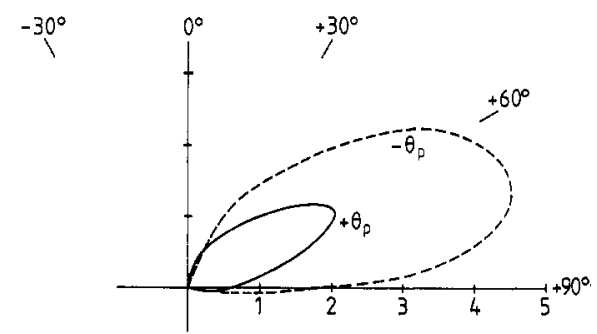

$$
\begin{aligned}
& \left|2 \pi \sin \theta_{e}\right| d^{3} \sigma / d e_{e} d \Omega_{p} d \Omega_{e}\left(10^{-9} \mathrm{~cm}^{2} e V^{-1} s \Gamma^{-2}\right) \\
& \mathrm{U}^{33+}+\mathrm{Ar} \longrightarrow \mathrm{U}^{33+}+\mathrm{Ar}^{4+}+4 \mathrm{e} \\
& 1.4 \mathrm{MeV} \mathrm{U}^{-1} \\
& \theta_{p}= \pm 0.000057^{\circ} \\
& E_{0}=150 \mathrm{eV} \text {. }
\end{aligned}
$$

Figure 9. Theoretical triply differential cross sections for ionised electrons of $150 \mathrm{eV}$ from the $1.4 \mathrm{MeVu}^{-1} \mathrm{U}^{33+}+\mathrm{Ar}$ collision system. The scattering of the projectile ion was $1 \times$ $10^{-6} \mathrm{rad}$. The plus and minus scattering angles shown on the figure are for positive and negative deflections of the projectile.

\section{Conclusions}

Absolute differential scattering cross sections $\mathrm{d} \sigma / \mathrm{d}\left(p_{\mathrm{R} \perp} / p_{0}\right)$ for collisions of highly charged, high-energy heavy ions with $\mathrm{Ne}$ atoms at very small projectile scattering 
angles could be obtained by applying a new experimental technique, namely measuring the transverse recoil-ion momentum instead of that of the projectile. The data are found to be strongly dependent on the projectile's initial charge state.

From the comparison with theoretical $\mathrm{d} \sigma / \mathrm{d}\left(p_{\mathrm{P}_{\perp}} / p_{0}\right)=\mathrm{d} \sigma / \mathrm{d} \theta$ results obtained using the $n$ CTMC approach, it becomes evident that the deduced experimental $\theta=\left(p_{\mathrm{R} \perp} / p_{0}\right)$ should be in good agreement with directly measured projectile scattering angles at large $\theta$. For small projectile scattering angles, however, the theory predicts a strong influence of the target electron dynamics on the momentum balance of all involved particles. However, the degree of information gained by applying this new technique is equivalent to the one obtained by measuring projectile scattering angles. The experimental $\mathrm{d} \sigma / \mathrm{d}\left(p_{\mathrm{R} \perp} / p_{0}\right)$ are in good agreement with the theoretical results when the differential cross sections are calculated from the recoil-ion transverse momenta. Large deviations of the measured data, however, can be observed by comparing with theoretical values obtained from the projectile's final transverse momentum. From this comparison we conclude that the theoretically predicted anisotropic emission of highenergy electrons as well as the polarisation of the target electron cloud strongly influence the kinematics of the heavy particles. This conclusion is underlined by the good agreement between experimental and theoretical $\delta$-electron energy distributions for the $1.4 \mathrm{MeV} \mathrm{u}^{-1} \mathrm{U}^{32+}$ on $\mathrm{Ne}$ collision system (Schmidt-Böcking et al 1988). Considering the very high energy and the large nuclear mass of the projectile this result is completely unexpected.

For the further investigation of the dynamics of such collisions, the accuracy of the recoil-ion momentum determination for very distant collisions can be improved by reducing the influence of the target thermal motion. The development of a spectrometer with cooled target $(20 \mathrm{~K})$ is in progress.

Furthermore, coincidence measurements between momentum and charge-state analysed recoil ions and momentum analysed electrons become desirable since then, for singly charged recoil ions the dynamics of the collision process will be fully determined. From such experiments, which seem to be feasible, we can test the challenging theoretical prediction of 'rainbow scattering' (negative deflection angles of the projectile), which may occur in very distant collisions of high-energetic, highly charged heavy projectiles with atomic targets.

\section{Acknowledgments}

The experimental work was supported by Bundesministerium für Forschung and Technologie (BMFT: 060F/73) and GSI Darmstadt, FRG. The theoretical calculations (REO) have been supported by the Office of Fusion Energy of the US Department of Energy and an award from the Alexander von Humboldt foundation of the FRG.

\section{References}

Horbatsch M 1986 Z. Phys. D $1337-45$

Kelbch S, Cocke C L, Hagmann S, Horbatsch M, Kelbch C, Koch R and Schmidt-Böcking H 1988 Phys. Lett. 127 92-5

Kelbch S, Schmidt-Böcking H, Ullrich J, Schuch R, Justianiano E, Ingwersen H and Cocke C L $1984 Z$. Phys. A 317 9-15

McGuire J H and Weaver L 1977 Phys. Rev. A 16 41-7 
Olson R E 1988 Electronic and Atomic Collisions ed. H B Gilbody, W R Newell, F H Read and A C H Smith (Amsterdam: Elsevier) pp 271-85

Olson R E, Gray T J, Berry H G, Hale E B and Irby V D 1987a Phys. Rev. Lett. 59 36-9

Olson R E, Ullrich J and Schmidt-Böcking H 1987b J. Phys. B: At. Mol. Phys. 20 L809-14

Proc. Int. Conf. on Highly Charged Ions (Groningen) 19861987 Nucl. Instrum. Methods. B 23

Schmidt-Böcking H, Schmidt S, Euler J, Kelbch C, Koch R, Kraft G, Olson R, Mann R and Hagmann S 1988 GSI Report 04-88 pp 4-8

Schuch R, Schöne H, Miller P D, Krause H F, Dittner P F, Datz S and Olson R 1988 Phys. Rev. Lett. 60 925-8

Ullrich J, Horbatsch M, Dangendorf V, Kelbch S and Schmidt-Böcking H 1988a J. Phys. B: At. Mol. Opt. Phys. 21 611-24

Ulirich J, Schmidt-Böcking H and Kelbch C 1988b Nucl. Instrum. Methods A 268 216-24 